\title{
Clinical effects of rectal retractor application in prostate cancer radiotherapy
}

\author{
Amir Mohammad Arefpour ${ }^{1+}$, Mahshid Abbasi $^{1+} * \mathbb{D}$, Seied Rabi Mahdavi ${ }^{2,3}$, Mahdiyeh Shafieesabet ${ }^{1^{*}}$ (D) Pedram Fadavi $^{1}$ \\ Received: 24 Aug 2020 \\ Published: 31 May 2021
}

\begin{abstract}
Background: Radiation-induced rectal toxicities remain as a major risk during prostate radiotherapy. One approach to the reduction of rectal radiation dose is to physically increase the distance between the rectal wall and prostate. Therefore, the aim of this study was to evaluate whether the application of the rectal retractor (RR) can reduce rectal dose and toxicity in prostate cancer 3-dimensional conformal radiotherapy (3D-CRT).

Methods: Overall, 36 patients with localized prostate cancer were randomized into the 2 groups, 18 patients with RR in-place and 18 without RR. All patients underwent planning computed tomography (CT). Patients were treated with 70 Gy in 35 fractions of 3D-CRT. In the RR group, RR was used during cone-down 20 treatment fractions. Acute and late gastrointestinal (GI) toxicities were assessed using EORTC/RTOG scoring system weekly during radiotherapy, 3, and 12 months after treatment. Device-related events were recorded according to CTCAE version 4.0. Patient characteristics, cancer differences, and dosimetric data for the RR and non-RR groups were compared using a Man-Whitney U test for continuous variables, and Fisher exact test for categorical data. The EORTC/RTOG scores for the 2 groups were compared using Fisher exact test. A P value $<0.05$ was considered statistically significant.

Results: A RR significantly reduced mean dose (Dmean) to the rectum as well as rectal volume receiving 50\% to 95\% (V50-95\%) of prescribed dose. The absolute reduction of rectal Dmean was 10.3 Gy. There was no statistically significant difference in acute GI toxicity between groups during treatment or at 3 months. At 12 months, 2 patients in the RR group and 9 in the control group experienced late grade $\geq 1$ GI toxicity ( $\mathrm{p}=0.027$ ). No patients in the RR group reported late grade $\geq 2$ GI toxicity, whereas 3 patients in the control group experienced late grade 2 GI toxicity. In the RR group, 6 patients reported grade 1 rectal discomfort and pain according to CTCAE version 4.0.
\end{abstract}

Conclusion: The application of the RR showed a significant rectum sparing effect, resulting in substantially reducing late GI toxicity.

Keywords: Prostate cancer, Radiotherapy, Rectal toxicity, Rectal retractor

Conflicts of Interest: None declared

Funding: This study was partially funded by Iran University of Medical Sciences, Tehran, Iran (grant number 97-01-30-32988).

*This work has been published under CC BY-NC-SA 1.0 license.

Copyright $₫$ Iran University of Medical Sciences

Cite this article as: Mohammad Arefpour A, Abbasi M, Rabi Mahdavi S, Shafieesabet M, Pedram Fadavi. Clinical effects of rectal retractor application in prostate cancer radiotherapy. Med J Islam Repub Iran. 2021 (31 May);35:69. https://doi.org/10.47176/mjiri.35.69

\section{Introduction}

Radiotherapy has a well-established role for managing localized prostate cancer, but the implementation of doseescalated prostate radiotherapy is limited due to rectal toxicity $(1,2)$. Even with improv ed prostate radiotherapy

Corresponding author: Dr Mahshid Abbasi, abbasi.m05@iums.acl.com DrMahdiyeh Shafieesabet, shafieesabet.m@iums.ac.com.com

\footnotetext{
+These authors contributed equally to this work.

1. Department of Radiation Oncology, School of Medicine, Iran University of Medical Sciences, Tehran, Iran

2. Department of Medical Physics, School of Medicine, Iran University of Medical Sciences, Tehran, Iran

3. Radiation Biology Research Center, Iran University of Medical Sciences, Tehran, Iran
}

techniques such as 3-dimensional conformal radiotherapy (3D-CRT) and intensity modulated radiotherapy (IMRT), radiation-induced rectal toxicities remain as a major risk owing to the close proximity of the rectum and prostate

$\uparrow$ What is "already known" in this topic:

Radiation-induced rectal toxicities limit dose-escalated prostate radiotherapy due to the close proximity of the rectum and prostate. To date, several studies showed that using a rectal retractor (RR) can significantly reduce rectal wall dose during prostate radiotherapy.

\section{$\rightarrow$ What this article adds:}

The present study is the first to evaluate the effect of the RR on rectal toxicity. The results indicated that the application of RR can significantly reduce late grade $\geq 1$ rectal toxicity. 
gland $(2,3)$.

One approach to the reduction of rectal radiation dose is to physically increase the distance between the rectal wall and prostate, thereby displacing the rectum from the high dose regions. This can be achieved with insertion of the rectal retractor (RR) into the rectum to displace rectal walls from the prostate (4-9). The RR system can retract the rectum dorsally, resulting in a significant reduction of rectal high dose irradiation volume $(4,6,7)$. In the previous study, it has been reported that the RR is well tolerated by patients (7). The published studies on the RR application during prostate radiotherapy focus on the impact of this device on the rectal dosimetry and the reduction of prostate motion $(4-7,10,11)$. The application of the RR can provide a great opportunity to reduce intrafractional prostate motion $(10,11)$, allowing for a tight planning target volume (PTV) margin. Although previous studies have demonstrated that using a RR can significantly reduce the rectal wall doses $(6,7)$, data on clinical outcomes of this method is scarce. Therefore, the purpose of this study was to report the clinical outcomes of prostate radiotherapy with a fractionation schedule of 70 Gy in 35 fractions over 7 weeks, when a RR was employed. Our endpoints were the comparison of rectal radiation doses achieved, acute gastrointestinal (GI) toxicity both during treatment and 3 months after treatment, and late GI toxicity.

\section{Methods}

\section{Setting and Patients}

A single-institution, randomized, pilot study of the RR application was approved by the ethic committee of Iran University of Medical Sciences, Tehran, Iran. Informed consent was obtained from all study participants.

Patients with biopsy-confirmed diagnosis of prostate cancer, with stage $\mathrm{T} 1$ or $\mathrm{T} 2$, a Gleason score $\leq 7$, and a prostate-specific antigen (PSA) level of $\leq 20 \mathrm{ng} / \mathrm{mL}$ were included in the present study to treat with 3D-CRT. Patients with metastatic disease, prior history of pelvic irradiation, prostatectomy, rectal or gastrointestinal surgery, and anorectal diseases such as hemorrhoids, rectal fissure, and fistula were excluded from the study.

\section{Rectal Retractor}

The instruction for placing RR has been described previously $(4,7)$. In brief, a RR system consists of 3 main parts, including rectal rod, vertical locking column, and carbon fiber baseplate, as shown in Figure 1. The plastic rectal rod is inserted into rectum and extended beyond the prostate and seminal vesicles. Then, this rod is connected to the vertical locking column attached to the carbon fiber baseplate. When the vertical docking column is displaced toward the bottom, the rectal rod displaces the rectum posteriorly. In our study, a physician inserted the rectal rod into the rectum in both planning computed tomography (CT) and daily treatment. For hygienic reasons, a rectal rod was covered by a disposable condom in each fraction and sterilized after each use of device. To facilitate daily insertion of the rectal rod lubricant or lidocaine jelly was used. The diameter of the rectal rod is $1.5 \mathrm{~cm}$.

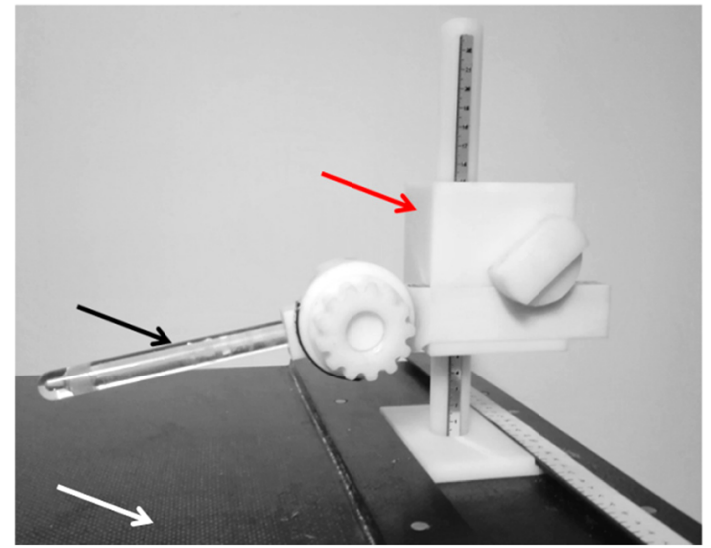

Fig. 1. The rectal retractor system; rectal rod (black arrow), vertical locking column (red arrow), and carbon fiber baseplate (white arrow).

\section{Planning and Treatment}

Prior to planning CT and daily treatment, all patients were instructed to have a full bladder and empty rectum. A comfortably full bladder was achieved by voiding bladder and then drinking $500 \mathrm{~mL}$ water, 30 to 40 minutes before CT-planning, and each treatment session. Laxative was prescribed before simulation. All patients underwent CT-scan in the supine position with a slice thickness of 3 $\mathrm{mm}$. For patients in the RR group, 2 planning CT scans was performed, 1 at the beginning of treatment with the RR and another after the $20^{\text {th }}$ treatment session without RR (Fig. 2), as RR was employed in cone-down 20 treatment fractions. In the current study, RR was used only in 20 fractions because the effect of the RR on anal sphincter function has not been evaluated. Also, daily insertion of the RR may lead to anal irritation and increase acute anorectal toxicities.

All CT images were imported into the Core-PLAN (version 3.5.0.5, Seoul C \& J Co., Seoul, South Korea) 3D treatment planning system for 3D-CRT treatment planning. The prostate gland and entire of seminal vesicles were considered as the clinical target volume (CTV) and contoured by responsible radiation oncologist. The PTV was generated by adding an isotropic margin of $10 \mathrm{~mm}$ around the CTV, except $8 \mathrm{~mm}$ in the posterior direction. In addition, the rectum, bladder, and femoral heads were contoured as organ at risks for all patients. The rectum was contoured from the anal verge superiorly to the rectosigmoid junction. All patients were treated with a linear accelerator (Siemens ONCOR, Germany) and 3D-CRT was delivered with a $15 \mathrm{MV}$ photons of 5 static fields using multileaf collimators. A total dose of 70 Gy using daily fraction dose of 2 Gy was prescribed to the prostate and the PTV. At least $95 \%$ of the PTV had to receive at least $95 \%$ of the prescription dose, with maximum dose $\leq$ $107 \%$ of the prescription. Dose-volume constraints were defined according to criteria proposed by the QUANTEC study for the rectum, bladder, and femoral heads. 


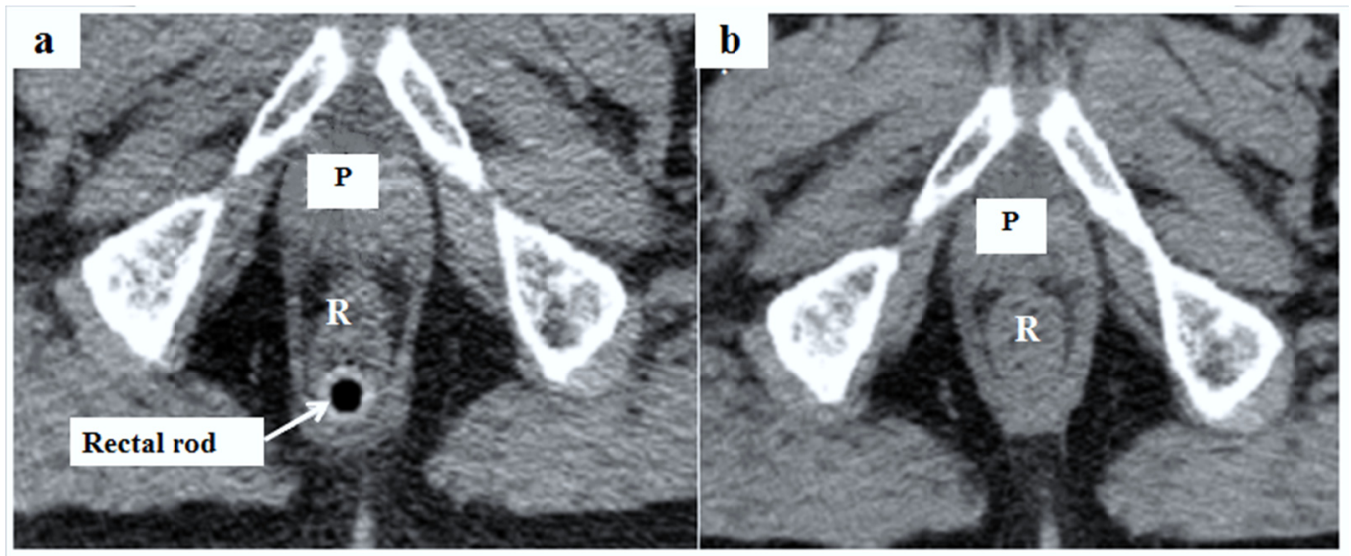

Fig. 2. CT-scan with a rectal retractor in-place (a) and without a rectal retractor (b) for a representative patient. The white arrow shows the rectal rod of the rectal retractor system.

\section{Toxicity Assessment}

Acute and late radiotherapy-induced GI toxicity were recorded according to the European Organization for Research and Treatment of Cancer/ Radiation Therapy Oncology Group (EORTC/RTOG) criteria. Patients were evaluated weekly during 3D-CRT, 1-, 3-, 9-, and 12month follow-up visits. Acute side effects were assessed weekly within irradiation and up to 3 months after the end of radiotherapy. Late GI toxicity was similarly evaluated at visits 9 and 12 months after 3D-CRT completion. Device-related events were recorded through the 12 months follow-up visit using Common Terminology Criteria for Adverse Events (CTCAE) version 4.0.

\section{Statistical Analysis}

Statistical analysis was performed using the SPSS 16.0 software (SPSS Inc). The normal distribution of each variable was investigated using Shapiro-Wilk test. Patient characteristics, cancer differences, and dosimetric data for the RR and non-RR groups were compared using a ManWhitney U test for continuous variables, and Fisher exact test for categorical data. The EORTC/RTOG scores for the 2 groups were compared using the Fisher exact test. A $\mathrm{P}$ value $<0.05$ was considered statistically significant.

\section{Results}

Between November 2018 and October 2019, a total of 36 patients were included within this study, 18 in the RR group and 18 in the control group. There were no statistically significant differences between groups in demographics or baseline tumor characteristics, as summarized in Table 1.

Using a RR significantly increased the perirectal distance $(p<0.001)$. The mean distance between the CTV and anterior rectal wall at the midgland level was $4.7 \pm 2.0 \mathrm{~mm}$ in the RR group and $2.5 \pm 1.7 \mathrm{~mm}$ in the control group. Table 2 shows the mean DVH values for the PTV and rectum in the RR and control groups. There was no statistically significant difference in the PTV coverage between groups; and the mean values for mean dose $\left(\mathrm{D}_{\text {mean }}\right)$ to the PTV for the RR and control groups were $70.7 \pm 0.8 \mathrm{~Gy}$ and $70.5 \pm 0.6$ Gy, respectively $(p=0.424)$. The application of the RR significantly reduced rectal $\mathrm{D}_{\text {mean }}$, with an absolute reduction of $10.3 \mathrm{~Gy}$, as observable in Table 2. A significant reduction to the rectal dose in the high-dose and intermediate-dose levels was seen with 3D-CRT after the $\mathrm{RR}$ application (Table 2). The volume of rectum receiving $100 \%$ prescribed dose was comparable in all plans $(\mathrm{p}=0.267)$.

Six out of 18 patients with a RR in-place experienced

Table 1. Patient and tumor characteristics $(\mathrm{n}=36)$

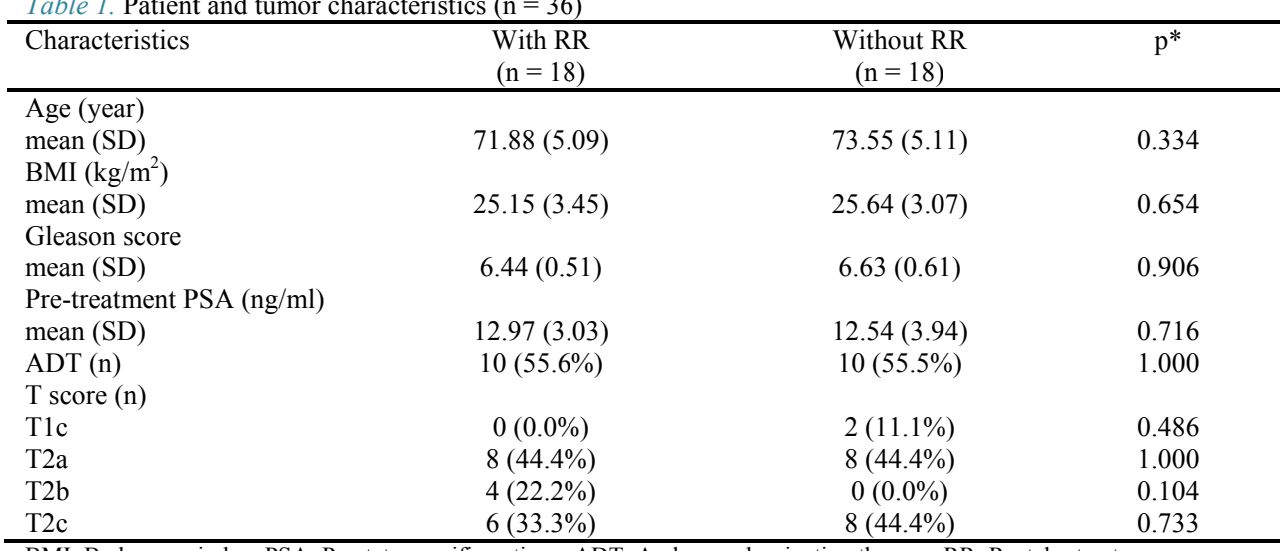

BMI: Body mass index; PSA: Prostate-specific antigen; ADT: Androgen deprivation therapy; RR: Rectal retractor

* P-value $<0.05$ was considered statistically significant. 


\begin{tabular}{|c|c|c|c|c|}
\hline & DVH parameter & $\begin{array}{l}\text { With RR } \\
(\mathrm{n}=18)\end{array}$ & $\begin{array}{c}\text { Without RR } \\
(\mathrm{n}=18)\end{array}$ & $\mathrm{p}^{*}$ \\
\hline \multicolumn{5}{|l|}{ PTV } \\
\hline & $\mathrm{D}_{\text {mean }}(\mathrm{Gy})$ & $70.7 \pm 0.8$ & $70.5 \pm 0.6$ & 0.424 \\
\hline & $\mathrm{V}_{95 \%}(\%)$ & $98.09 \pm 1.33$ & $98.02 \pm 1.42$ & 0.874 \\
\hline \multicolumn{5}{|l|}{ Rectum } \\
\hline & $\mathrm{D}_{\text {mean }}(\mathrm{Gy})$ & $33.91 \pm 2.89$ & $44.21 \pm 3.90$ & $<0.001$ \\
\hline & $\mathrm{D}_{\max }(\mathrm{Gy})$ & $69.92 \pm 0.95$ & $70.03 \pm 0.96$ & 0.735 \\
\hline & $\mathrm{D}_{\min }(\mathrm{Gy})$ & $2.23 \pm 0.63$ & $2.11 \pm 0.52$ & 0.538 \\
\hline & $\mathrm{D}_{30 \%}(\mathrm{~Gy})$ & $41.58 \pm 4.81$ & $53.31 \pm 4.54$ & $<0.001$ \\
\hline & $\mathrm{D}_{50 \%}$ (Gy) & $26.82 \pm 10.63$ & $38.88 \pm 2.73$ & $<0.001$ \\
\hline & $\mathrm{V}_{50 \%}(\%)$ & $43.26 \pm 5.64$ & $56.07 \pm 3.28$ & $<0.001$ \\
\hline & $\mathrm{V}_{60 \%}(\%)$ & $34.18 \pm 5.25$ & $46.51 \pm 3.44$ & $<0.001$ \\
\hline & $\mathrm{V}_{70 \%}(\%)$ & $26.96 \pm 5.90$ & $37.07 \pm 4.95$ & $<0.001$ \\
\hline & $\mathrm{V}_{80 \%}(\%)$ & $20.19 \pm 4.95$ & $32.23 \pm 6.27$ & $<0.001$ \\
\hline & $\mathrm{V}_{90 \%}(\%)$ & $14.37 \pm 4.17$ & $25.23 \pm 4.42$ & $<0.001$ \\
\hline & $\mathrm{V}_{95 \%}(\%)$ & $10.10 \pm 3.64$ & $19.02 \pm 3.28$ & $<0.001$ \\
\hline & $\mathrm{V}_{100 \%}(\%)$ & $0.88 \pm 0.88$ & $1.21 \pm 0.88$ & 0.267 \\
\hline
\end{tabular}

CTV: Clinical target volume; PTV: Planning target volume; Dmean: Mean dose to the rectum (or target volume);

Dmax: Maximum dose to the rectum; Dmin: Minimum dose to the rectum; Dx\% dose to $\mathrm{x} \%$ of the rectal wall

volume, $\mathrm{Vx} \%$ : Volume of the rectum (or target volume) receiving $\mathrm{x} \%$ of prescribed dose); RR: Rectal retractor.

* P-value $<0.05$ was considered statistically significant.

Table 3. Comparison of gastrointestinal toxicity in patients treated with and without rectal retractor using the RTOG/EORTC scoring criteria

\begin{tabular}{llccc}
\hline \multicolumn{5}{c}{ Grade $\geq 1$} \\
& Follow-up period & $\begin{array}{c}\text { With RR } \\
(\mathrm{n}=18)\end{array}$ & $\begin{array}{c}\text { Without RR } \\
(\mathrm{n}=18)\end{array}$ & $\mathrm{p}^{*}$ \\
\hline Acute & During RT & $10(55.5 \%)$ & $8(44.4 \%)$ & 0.740 \\
& $1-3$ months after RT & $6(33.3 \%)$ & $10(55.5)$ & 0.315 \\
Late & $9-12$ months after RT & $2(11.1 \%)$ & $9(50.0 \%)$ & 0.027 \\
\hline
\end{tabular}

RT: Radiotherapy; RR: Rectal retractor

* P-value $<0.05$ was considered statistically significant.

grade 1 rectal discomfort and pain as device-related events according to the CTCAE v.4.0. All of these events were self-limited and resolved with no additional treatment during cone-up 15 fractions without the RR in-place. These events occurred approximately after the $15^{\text {th }}$ treatment fraction with a RR.

The radiation-induced GI toxicity data are outlined in Table 3. During the radiotherapy period and the following 3 months, there was no statistically significant difference in acute grade $\geq 1$ GI toxicity between the RR and control groups, as observable in Table 3 . Acute grade 2 GI toxicity was reported in the 8 patients during treatment, 4 in the RR group, and 4 in the control group. No significant difference was found in acute grade 2 GI toxicity between groups ( 2 patients in the RR group and 4 patients in the control group $(p=0.658))$. Overall, the rate of acute grade 1-2 toxicity was similar between the 2 groups. Acute GI toxicities mostly were in the form of increased stool frequency, diarrhea, and rectal mucous discharge. The increased stool frequency was resolved without the use of medication. Loperamide was used to resolve diarrhea and rectal mucous discharge. No acute grade 3 GI toxicity was reported in either arm.

As shown in Table 3, at 12 months, 2 patients in the RR group and 9 patients in the control group experienced late grade $\geq 1$ GI toxicity $(p=0.027)$. No late grade $\geq 2$ GI toxicity was reported in the RR group. In the control group, late grade 2 GI toxicity was reported in the 3 patients, with no patients experiencing late grade 3 GI toxicity.

\section{Discussion}

Our data indicate that using a RR significantly reduces rectal dose volume parameters and consistently results in reducing late grade $\geq 1$ GI toxicity. No significant difference was found in acute GI toxicity between patients in the RR and control groups.

The dosimetric advantage and sparing the rectum from high-dose areas have been clearly demonstrated in the current study and in prior studies evaluating a RR application (4-7). From our data, it can be seen that the application of the RR significantly improves rectum dosimetry. As shown in Figure 2, when the rectum is retracted by RR, its shape changes, resulting in reducing rectal wall volume in THE high-dose region. As observable in Table 2, using a RR significantly reduces rectal $D_{\text {mean }}$ and $V_{50-95 \%}$. These data are in good agreement with previous study that showed the application of a RR resulted in an absolute reduction of $13.6 \mathrm{~Gy}$ in rectal wall $\mathrm{D}_{\text {mean }}$ as well as a relative reduction more than $50 \%$ in rectal wall $\mathrm{V}_{50-75 \text { Gy }}$ during image-guided dose-escalated prostate 3D-CRT, with a total dose of $80 \mathrm{~Gy}$ in 40 fractions (7). In the present study, the maximum dose to the rectum $\left(\mathrm{D}_{\max }\right)$ was reduced in the RR group but wasi not statistically significant. However, a study by Mahdavi et al showed that the RR application results in a significant decrease of rectal wall $D_{\max }(7)$. A possible cause of this discrepancy can be attributed to the size of the CTV-PTV margins in 2 studies. Mahdavi et al added an isotropic margin of $5 \mathrm{~mm}$ around the CTV, because they used gold fiducial marker-based image-guided radiotherapy technique (7), whereas we 
applied a margin of $10 \mathrm{~mm}$ around the CTV, except posteriorly which was $8 \mathrm{~mm}$. In the prostate radiotherapy, it has been demonstrated that daily pretreatment imaging using implanted fiducial markers into the prostate can significantly reduce the PTV margins, allowing for greater sparing of the rectum or bladder (12).

In the present study, it was found that the rate of acute grade $\geq 1$ GI toxicity is similar between the groups. A possible cause that can elucidate this issue is toxicity secondary to unintended irradiation to small bowel or sigmoid colon. Recent evidence suggests that acute bowel toxicity can be associated with radiation doses between 20 Gy and 40 Gy to these tissues $(13,14)$. Although the RR displaces the rectum from high-dose levels, small bowel or sigmoid colon receives these above-mentioned low dose levels. Besides, daily physical insertion of the RR along with daily irradiation can increase sensitivity of rectal mucous, resulting in anal and rectal irritation and proctitis. In fact, some acute rectal toxicity in the RR group may not be from radiotherapy, but from the RR itself. With long follow-up time, it has been shown that GI toxicity in the RR group was found to be less than that in the control group, as observable in Table 3. These results could be largely associated with the RR application. Our data indicate that device-related events are slight and limited to grade 1 rectal discomfort and pain. We did not find severe procedure-related toxicities such as rectal bleeding, perforation, et cetera. The patients reported that the placement of the RR was discomfort, but easily tolerable.

Several other researchers used the endorectal balloons (ERBs) and hydrogel spacer for increasing space between the prostate and rectal wall, thereby decreasing rectal radiation doses (15-22). Table 4 summarizes studies evaluating the impact of different rectal displacement devices
(RDDs) on rectal toxicity during prostate external beam radiotherapy (EBRT). As displayed in Table 4, the rate of acute GI toxicity remains to be relatively high, when RDDs are employed. In contrast, the rate of late rectal toxicity, especially late grade $\geq 2$ toxicity remarkably reduces with the application of the RDDs. Of note, the implantation of hydrogel spacer compared to ERBs or RR appears to be more effective in reducing late rectal toxicity (19-21). In a prospective multicenter randomized phase III trial, Hamstra et al have reported that the rate of late grade $\geq 1$ rectal toxicity was $2 \%$ in the patients with hydrogel spacer in situ (21). From a radiobiological point of view, the rectum is a late responding normal tissue (23); therefore, long-term follow-up duration is required to clarify the effect of the RR on reducing rectal toxicity.

Several studies have been published about the effect of RDDs on prostate motion (10, 11, 24-26). Several groups of researchers evaluated the impact of the ERBs and RR on reducing intrafractional prostate motion using Calypso 4D real-time tracking system, kilovoltage intrafraction monitoring, and cine-magnetic resonance imaging (MRI) and showed that the ERBs and RR can significantly decrease the intrafractional prostate motion $(10,11,25,26)$, but clinical outcomes of this prostate motion reduction are unclear. It should be noted that the aim of managing prostate movement is the rectal toxicity reduction; therefore, there is a need for further research. Although the injection of hydrogel spacer does not reduce prostate motion (24, $27,28)$, the results of clinical trials have demonstrated that this method can significantly reduce radiotherapy induced-rectal injuries, leading to long-term improvements in patient's quality of life $(20,21,29)$.

The RR offers several advanttages compared to the ERB. First, the RR does not push the anterior rectal wall to-

Table 4. The comparison of gastrointestinal toxicity in patients treated with various types of rectal displacement devices

\begin{tabular}{|c|c|c|c|c|c|c|c|c|c|}
\hline \multirow[t]{2}{*}{ Study } & \multirow{2}{*}{ RT technique } & \multirow{2}{*}{$\begin{array}{c}\text { No. of } \\
\text { patients }\end{array}$} & \multirow[t]{2}{*}{ RDDs } & \multirow{2}{*}{$\begin{array}{l}\text { Rectal dose } \\
\text { metric }\end{array}$} & \multirow{2}{*}{$\begin{array}{c}\text { Follow up/ } \\
\text { scoring criteria }\end{array}$} & \multicolumn{2}{|c|}{ Acute toxicity } & \multicolumn{2}{|c|}{ Late toxicity } \\
\hline & & & & & & $\mathrm{G} \geq 1$ & $\mathrm{G} \geq 2$ & $\mathrm{G} \geq 1$ & $\mathrm{G} \geq 2$ \\
\hline Teh et al./ $2001[15]$ & $70 \mathrm{~Gy}$ in $35 \mathrm{~F} / \mathrm{IMRT}$ & 100 & ERB & $\mathrm{D}_{\text {mean }}(\mathrm{Gy}): 35.3$ & $\begin{array}{l}10.1 \text { months / } \\
\text { RTOG }\end{array}$ & $17 \%$ & $6 \%$ & NR & NR \\
\hline Goldner et al./ 2006 [16] & $\begin{array}{l}70 \text { Gy in } 35 \mathrm{~F} \text { or } 74 \\
\text { Gy in } 37 \mathrm{~F} / 3 \mathrm{DCRT}\end{array}$ & 429 & ERB & NR & $\begin{array}{c}\text { Acute only/ } \\
\text { EORTC/RTOG }\end{array}$ & $48.5 \%$ & $17.5 \%$ & NR & NR \\
\hline Goldner et al./ 2007 [17] & $\begin{array}{l}70 \mathrm{~Gy} \text { in } 35 \mathrm{~F} \text { or } 74 \\
\text { Gy in } 37 \mathrm{~F} / 3 \mathrm{DCRT}\end{array}$ & 166 & ERB & NR & $\begin{array}{c}40 \text { months/ } \\
\text { EORTC/RTOG }\end{array}$ & NR & NR & $42 \%$ & $31 \%$ \\
\hline Cho et al./ 2009 [18] & $\begin{array}{c}70.2 \mathrm{~Gy} \text { in } 39 \mathrm{~F} / \\
\text { 3DCRT }\end{array}$ & 35 & ERB & $\mathrm{D}_{\text {mean }}(\mathrm{Gy}): 43.5$ & $\begin{array}{l}\text { Acute only/ } \\
\text { RTOG }\end{array}$ & $57 \%$ & $17 \%$ & NR & NR \\
\hline Uhl et al./ 2013 [19] & $78 \mathrm{~Gy}$ in $39 \mathrm{~F} / \mathrm{IMRT}$ & 52 & HS & NR & $\begin{array}{c}12 \text { months/ } \\
\text { RTOG/EORTC }\end{array}$ & $52 \%$ & $12 \%$ & $7 \%$ & $0 \%$ \\
\hline Whalley et al./ 2016 [20] & $\begin{array}{c}80 \mathrm{~Gy} \text { in } 40 \mathrm{~F} / \\
\text { IG-IMRT or VMAT }\end{array}$ & 30 & HS & $\begin{array}{l}\mathrm{D}_{\text {mean }}(\mathrm{Gy}): 28 \\
\mathrm{~V}_{\text {70Gy }}(\%): 3.7\end{array}$ & $\begin{array}{l}28 \text { months/ } \\
\text { RTOG }\end{array}$ & $43 \%$ & $0 \%$ & $19.9 \%$ & $3.3 \%$ \\
\hline Hamstra et al./ 2017 [21] & $\begin{array}{c}79.2 \text { Gy in } 44 \text { F/ IG- } \\
\text { IMRT }\end{array}$ & 149 & HS & $\mathrm{V}_{70 \mathrm{~Gy}}(\%): 2$ & $\begin{array}{l}37 \text { months/ } \\
\text { CTCAE v.4.0. }\end{array}$ & NR & NR & $2 \%$ & $0 \%$ \\
\hline Current study & $\begin{array}{c}70 \mathrm{~Gy} \text { in } 35 \mathrm{~F} / \\
\text { 3DCRT }\end{array}$ & 18 & $\mathrm{RR}$ & $\begin{array}{c}\mathrm{D}_{\text {mean }}(\mathrm{Gy}): 33.9 \\
\mathrm{~V}_{100 \%}(\%): 0.9\end{array}$ & $\begin{array}{l}12 \text { months/ } \\
\text { EORTC/RTOG }\end{array}$ & $33.3 \%$ & $22.2 \%$ & $11.1 \%$ & $0 \%$ \\
\hline
\end{tabular}

F: Fraction; IMRT: Intensity modulated radiotherapy; 3DCRT: Three-dimensional conformal radiotherapy; VMAT: Volumetric modulated arc radiotherapy; RDD: Rectal displacement device; ERB: Endorectal balloon; HS: Hydrogel spacer; RR: Rectal retractor; NR: Not reported; RTOG: Radiation Therapy Oncology Group; EORTC; European Organization for Research and Treatment of Cancer; CTCAE: Common Terminology Criteria for Adverse Event; G: Grade 
wards the prostate $(4,7)$, whereas studies have reported that using an ERB can result in increasing radiation dose to the anterior rectal wall (30). Unlike ERB, previous studies showed that using a RR can significantly reduce radiation dose to the anterior rectal wall $(7,31)$. Second, the daily positional reproducibility of the RR is high (6) because the rectal rod is connected into vertical locking column; therefore, the depth of the insertion of the rectal rod is identical during simulation and daily treatment. In contrast, there are variations in daily ERB placement, mostly due to error in the insertion depth (32). As a consequence, daily verification of the ERB position is necessary. Of note, using a RR does not change the shape of the prostate gland, as observable in Figure 2. Third, the application of a RR can increase interfractional rectum position (6); thereby reducing interfractional prostate motion, whereas studies have demonstrated the ERB cannot decrease interfractional prostate displacement (25).

It is worthwhile to mention that hydrogel spacer is injected into Denonvilliers' fascia, resulting in a clear space between the anterior rectal wall and prostate, with a mean separation of $10 \mathrm{~mm}(22,33)$. Our data show that the RR can increase the distance between the rectum and CTV. We measured the shortest distance between the anterior rectal wall and posterior border of the CTV at midgland level on CT images. Of note, CT-scan cannot well distinguish the prostate-rectum separation due to low soft tissue contrast. Therefore, the reliability of our results is low. Further study with MRI will be required to elucidate whether the retraction of the rectum can increase the distance between the anterior rectal wall and prostate. Hydrogel spacer compared to the ERB and RR has also been utilized in prostate brachytherapy with promising results (22, 33-35). A disadvantage of the ERB and RR compared to hydrogel spacer is that daily insertion of these devices is time-consuming, resulting in increasing in-room time (7), whereas hydrogel spacer is injected prior to CTplanning at the same time that fiducial markers are implanted (22). Patients with hemorrhoidal disease cannot use RR and ERB because the insertion of rectal rod and ERB into the rectum leads to burning and inflammation of the anus. In these patients, hydrogel spacer is useful. With regard to postprostatectomy radiotherapy, studies have showed that using an ERB does not significantly reduce anorectal wall doses because missing counterfort of the prostate in the postoperative setting can lead to pushing anterior rectal wall into the prostate bed (36). In contrast, the RR and hydrogel spacer can be used in the wellselected patients, resulting in a significant reduction of rectal wall doses during postprostatectomy radiotherapy $(4,37)$; however, further study will be required to determine whether this rectal dose reduction results in improving clinical outcomes.

It is important to consider the benefits of RDDs against the possible risks of complications related to procedure of these technologies. Our data show that RR is discomfort and can result in rectal pain. This is also true for ERB. The RR may be more discomfort compared to ERB, because the rectal rod is rigid, whereas rectal balloon is made of latex; therefore, it is flexible. On the other hand, although the rectal rod is rigid, residual stool in the rectum does not have any influence on the rectal rod, whereas residual gas and stool in the rectum can significantly reduce daily reproducibility of the ERB position (38). With regard to the implantation of hydrogel spacer, several cases of rectal perforation, bacterial prostatitis, rectal ulceration, perineal abscess, and rectal discomfort and pain were reported $(22,39)$; however, the rate of these complications is very low.

The effectiveness of a RR that retracts rectal walls and provides a reproducible rectal wall position has substantial clinical implications for prostate cancer radiotherapy, including hypofractionation, dose escalation, reirradiation, and postprostatectomy radiotherapy. The most important feature of RR in comparison to other techniques is its ability for doing real-time dosimetry to verify the absolute delivered dose in place. In addition, the RR may result in cost-saving for the healthcare system. Long-term clinical outcomes are required to better evaluate a possible benefit for patients undergoing prostate radiotherapy with a RR in-place.

\section{Conclusion}

The application of RR was feasible. The retraction of the rectum resulted in reducing rectal doses and the rates of late rectal toxicities. RR can be an effective device, especially with the advanced prostate radiotherapy techniques. Further study with large sample size is required to confirm clinical benefits from this device.

\section{Acknowledgement}

The authors are thankful to the staff of Firoozgar hospital (Tehran, Iran). This study was partially granted by research chancellor of Iran University of Medical Sciences, Tehran, Iran.

Research Involved Human Piarticipants and/or Animals

This study involved human participants, and it was conducted considering ethic responsibilities according to the World Medical Association and the Declaration of Helsinki.

\section{Ethical Approval}

The study was approved by the ethics committee of Iran University of Medical Sciences, Tehran, Iran. Ethics No. is IR.IUMS.FMD.REC.1396.9411188001.

\section{Informed Consent}

Informed consent was obtained from all individual participants prior to their inclusion in the study.

\section{Conflict of Interests}

The authors declare that they have no competing interests.

\section{References}

1. Viani GA, Stefano EJ, Afonso SL. Higher-Than-Conventional Radiation Doses in Localized Prostate Cancer Treatment: A Metaanalysis of Randomized, Controlled Trials. Int J Radiat Oncol Biol Phys. 2009;74:1405-18. 
2. Kuban DA, Tucker SL, Dong L, Starkschall G, Huang EH, Cheung MR, et al. Long-Term Results of the M. D. Anderson Randomized Dose-Escalation Trial for Prostate Cancer. Int J Radiat Oncol Biol Phys. 2008;70:67-74.

3.Zietman AL, Bae K, Slater JD, Shipley WU, Efstathiou JA, Coen JJ, et al. Randomized trial comparing conventional-dose with high-dose conformal radiation therapy in early-stage adenocarcinoma of the prostate: long-term results from proton radiation oncology group/american college of radiology 95-09. J Clin Oncol. 2010;28:1106-11.

4. Ghaffari H, Afkhami Ardekani M, Molana SH, Haghparast M, Sanei M, Mahdavi SR, et al. Application of rectal retractor for postprostatectomy salvage radiotherapy of prostate cancer: A case report and literature review. Clin Case Rep. 2019;7:2102-7.

5. Ghaffari H, Mahdavi S, Mofid B, Reiazi R. Rectal sparing using a rectal retractor during dose escalated prostate radiotherapy. Med Phys. 2018;45: E254.

6. Isacsson U, Nilsson K, Asplund S, Morhed E, Montelius A, Turesson I. A method to separate the rectum from the prostate during proton beam radiotherapy of prostate cancer patients. Acta Oncol. 2010;49:500-5.

7. Mahdavi SR, Ghaffari H, Mofid B, Rostami A, Reiazi R, Janani L. Rectal retractor application during image-guided dose-escalated prostate radiotherapy. Strahlenther Onkol. 2019;195:923-33.

8. Nicolae A, Davidson M, Easton H, Helou J, Musunuru H, Loblaw A, et al. Clinical evaluation of an endorectal immobilization system for use in prostate hypofractionated Stereotactic Ablative Body Radiotherapy (SABR). Radiat Oncol. 2015;10:122.

9. Ghaffari H. Rectal wall delineation in patients with a rectal displacement device in place during prostate cancer radiotherapy. J Radiat Oncol. 2019;8:103-4

10. de Leon J, Jameson MG, Rivest-Henault D, Keats S, Rai R, Arumugam S, et al. Reduced motion and improved rectal dosimetry through endorectal immobilization for prostate stereotactic body radiotherapy. Br J Radiol. 2019;92:20190056.

11. Legge K, Nguyen D, Ng JA, Wilton L, Richardson M, Booth J, et al. Real-time intrafraction prostate motion during linac based stereotactic radiotherapy with rectal displacement. J Appl Clin Med Phys. 2017; 18:130-6.

12. Ghaffari H, Navaser M, Mofid B, Mahdavi SR, Mohammadi R, Tavakol A. Fiducial markers in prostate cancer image-guided radiotherapy. Med J Islam Repub Iran. 2019;33:15.

13. Karsh LI, Gross ET, Pieczonka CM, Aliotta PJ, Skomra CJ, Ponsky LE, et al. Absorbable Hydrogel Spacer Use in Prostate Radiotherapy: A Comprehensive Review of Phase 3 Clinical Trial Published Data. Urology. 2018;115:39-44.

14. Sini C, Noris Chiorda B, Gabriele P, Sanguineti G, Morlino S, Badenchini $\mathrm{F}$, et al. Patient-reported intestinal toxicity from whole pelvis intensity-modulated radiotherapy: First quantification of bowel dose-volume effects. Radiother Oncol. 2017;124:296-301.

15. Teh BS, Mai WY, Uhl BM, Augspurger ME, Grant WH, III, Lu HH, et al. Intensity-modulated radiation therapy (IMRT) for prostate cancer with the use of a rectal balloon for prostate immobilization: acute toxicity and dose-volume analysis. Int J Radiat Oncol Biol Phys. 2001;49:705-12.

16. Goldner G, Geinitz H, Wachter S, Becker G, Zimmermann F, Wachter-Gerstner N, et al. 3-D Conformal radiotherapy of localized prostate cancer within an Austrian-German multicenter trial: a prospective study of patients' acceptance of the rectal balloon during treatment. Wien Klin Wochenschr. 2006;118:224-9.

17. Goldner G, Tomicek B, Becker G, Geinitz H, Wachter S, Zimmermann $\mathrm{F}$, et al. Proctitis after external-beam radiotherapy for prostate cancer classified by Vienna Rectoscopy Score and correlated with EORTC/RTOG score for late rectal toxicity: Results of a prospective multicenter study of 166 patients. Int J Radiat Oncol Biol Phys. 2007;67:78-83.

18. Cho JH, Lee CG, Kang DR, Kim J, Lee S, Suh CO, et al. Positional reproducibility and effects of a rectal balloon in prostate cancer radiotherapy. J Korean Med Sci. 2009;24:894-903.

19. Uhl M, van Triest B, Eble MJ, Weber DC, Herfarth K, De Weese TL. Low rectal toxicity after dose escalated IMRT treatment of prostate cancer using an absorbable hydrogel for increasing and maintaining space between the rectum and prostate: Results of a multiinstitutional phase II trial. Radiother Oncol. 2013;106:215-9.

20. Whalley D, Hruby G, Alfieri F, Kneebone A, Eade T. SpaceOAR
Hydrogel in Dose-escalated Prostate Cancer Radiotherapy: Rectal Dosimetry and Late Toxicity. Clin Oncol. 2016;28:e148-e54.

21. Hamstra DA, Mariados N, Sylvester J, Shah D, Karsh L, Hudes R, et al. Continued Benefit to Rectal Separation for Prostate Radiation Therapy: Final Results of a Phase III Trial. Int J Radiat Oncol Biol Phys. 2017:97:976-85.

22. Afkhami Ardekani M, Ghaffari H. Optimization of prostate brachytherapy techniques with polyethylene glycol-based hydrogel spacers: A systematic review. Brachytherapy. 2020;19:13-23.

23. Michalski JM, Gay H, Jackson A, Tucker SL, Deasy JO. Radiation dose-volume effects in radiation-induced rectal injury. Int J Radiat Oncol Biol Phys. 2010;76:S123-S9.

24. Juneja P, Kneebone A, Booth JT, Thwaites DI, Kaur R, Colvill E, et al. Prostate motion during radiotherapy of prostate cancer patients with and without application of a hydrogel spacer: a comparative study. Radiat Oncol. 2015;10:215.

25. Smeenk RJ, Louwe RJ, Langen KM, Shah AP, Kupelian PA, van Lin EN, et al. An endorectal balloon reduces intrafraction prostate motion during radiotherapy. Int $\mathrm{J}$ Radiat Oncol Biol Phys. 2012;83:661-9

26. Wang KK, Vapiwala N, Deville C, Plastaras JP, Scheuermann R, Lin $\mathrm{H}$, et al. A study to quantify the effectiveness of daily endorectal balloon for prostate intrafraction motion management. Int J Radiat Oncol Biol Phys. 2012;83:1055-63.

27. Ghaffari H, Navaser M, Refahi S. In regard to Cuccia et al.: impact of hydrogel peri-rectal spacer insertion on prostate gland intra-fraction motion during $1.5 \mathrm{~T}$ MR-guided stereotactic body radiotherapy. Radiat Oncol. 2020;15:199.

28. Afkhami Ardekani M, Ghaffari H, Navaser M, Zoljalali Moghaddam $\mathrm{SH}$, Refahi S. Effectiveness of rectal displacement devices in managing prostate motion: a systematic review. Strahlenther Onkol. 2020. https://doi.org/10.1007/s00066-020-01633-9.

29. Mariados N, Sylvester J, Shah D, Karsh L, Hudes R, Beyer D, et al Hydrogel Spacer Prospective Multicenter Randomized Controlled Pivotal Trial: Dosimetric and Clinical Effects of Perirectal Spacer Application in Men Undergoing Prostate Image Guided Intensity Modulated Radiation Therapy. Int J Radiat Oncol Biol Phys. 2015:92:971-7.

30. Elsayed H, Bölling T, Moustakis C, Müller S-B, Schüller P, Ernst I, et al. Organ Movements and Dose Exposures in Teletherapy of Prostate Cancer using a Rectal Balloon. Strahlenther Onkol. 2007;183:617-24.

31. Ghaffari H, Rostami A, Ardekani MA, Mofid B, Mahdavi SR. Rectal wall sparing effect of a rectal retractor in prostate intensity-modulated $\begin{array}{lllll}\text { radiotherapy. } J \text { Can } & \text { Res }\end{array}$ https://doi.org/10.4103/jert.JCRT 701 19.

32. Jones BL, Gan G, Kavanagh B, Miften M. Effect of endorectal balloon positioning errors on target deformation and dosimetric quality during prostate SBRT. Phys Med Biol. 2013;58:7995-8006.

33. Afkhami Ardekani M, Navaser M, Ghaffari H, Refahi S. Letter to the editor on: F. Campostrini et al. "Association between acute histopathological changes of rectal walls and late radiation proctitis following radiotherapy for prostate cancer". Strahlenther Onkol. 2020 https://doi.org/10.1007/s00066-020-01719-4

34. Ghaffari H. Re: Placement of SpaceOAR hydrogel spacer for prostate cancer patients treated with iodine-125 low-dose-rate brachytherapy. Int J Urol. 2020; 27:473

35. Ghaffari H, Mashoufi M, Refahi S, Ardekani MA. Letter to the editor regarding "Rectal spacing, prostate coverage, and periprocedural outcomes after hydrogel spacer injection during lowdose-rate brachytherapy implantation”. Brachytherapy. 2020;19:554-

36. Streller T, Rusch U, Herraiz Lablanca MD, Minneken I, Najafi Y, Shrestha B, et al. The effect of endorectal balloon on anorectal dose during postoperative volumetric arc radiotherapy of prostate cancer. Radiother Oncol. 2017;123:454-8.

37. Ghaffari $\mathrm{H}$. Is there a role for hydrogel spacer in post-prostatectomy radiotherapy setting? Radiol Med. 2019;124:1062-3.

38. Wang KK, Vapiwala N, Bui V, Deville C, Plastaras JP, Bar-Ad V, et al. The impact of stool and gas volume on intrafraction prostate motion in patients undergoing radiotherapy with daily endorectal balloon. Radiother Oncol. 2014;112:89-94.

39. Ghaffari H, Afkhami Ardekani M, Refahi S. In regard to 'What is the quality of hydrogel spacer insertions? and which patients will benefit? A literature review'. J Radiother Pract. 2020; 19:403-404. 